\title{
Malignant conversion of a solitary squamous cell papilloma in the trachea treated by radiotherapy: A case report
}

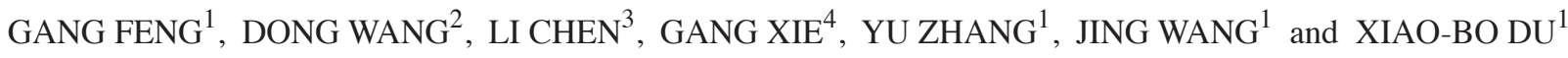 \\ Departments of ${ }^{1}$ Oncology, ${ }^{2}$ Surgery, ${ }^{3}$ General Practice and ${ }^{4}$ Pathology, \\ Mianyang Central Hospital, Mianyang, Sichuan 621000, P.R. China
}

Received May 29, 2014; Accepted February 10, 2015

DOI: $10.3892 / 01.2015 .3061$

\begin{abstract}
The present study described a rare case of malignant conversion of a solitary papilloma in the trachea, in addition to reviewing the current literature. A 54-year-old male presented with a cough, sputum and dyspnea. Chest computed tomography revealed a mediastinal soft-tissue mass in the trachea. A tumor biopsy, performed using a flexible bronchofiberscope, identified a squamous cell papilloma with moderate to severe atypical hyperplasia, as well as the formation of squamous cell carcinoma. Polymerase chain reaction analysis did not detect presence of human papilloma virus in the tumor. The patient was subsequently treated with radiotherapy and no evidence of recurrence was observed during a two-year follow-up period. To the best of our knowledge, this is the first study to describe the malignant conversion of a solitary papilloma in the trachea, which was subsequently treated with radiotherapy.
\end{abstract}

\section{Introduction}

Papillomatosis primarily presents as multiple lesions that arise in the oral cavity, larynx and vulva, and to a lesser extent, in segments of the trachea. It most frequently occurs in children or adolescents (1). Solitary papilloma is extremely rare and its occurrence in the trachea is even rarer, accounting for $<0.5 \%$ of all lung tumors (2). Tracheal papilloma is a benign neoplastic condition that is characterized by continuous papillomatous growth of the bronchial epithelium (3). In 3-5\% of patients, respiratory papillomas may undergo malignant degeneration to become a squamous cell carcinoma. The prognoses of these particular patients are relatively poor (4). Transformation is believed to be associated with infection by the human papilloma virus (HPV) and smoking. However, the mechanism underlying transformation is not fully understood (5).

Correspondence to: Dr Xiao-Bo Du, Department of Oncology, Mianyang Central Hospital, 12 Changjiaxiang, Mianyang, Sichuan 621000, P.R. China

E-mail: duxiaobo2005@126.com

Key words: trachea, papilloma, squamous cell carcinoma, radiotherapy
To the best of our knowledge, the present study is the first to describe the malignant conversion of a solitary tracheal papilloma into a squamous cell carcinoma in an adult patient who was subsequently treated by radiotherapy.

\section{Case report}

On August 20, 2011, a 54-year-old male was admitted to the Mianyang Central Hospital (Mianyang, China) with a cough, sputum and dyspnea that had been apparent for one month. The patient was a farmer and had been smoking $>20$ cigarettes per day for $>30$ years; however, the patient had not been exposed to other risk factors, including carcinogenic agents, immunosuppressants or radiation. The patient had been previously diagnosed with pneumonia and treated accordingly, until the symptoms of dyspnea worsened and hemoptysis developed.

A physical examination revealed that the patient had a temperature of $38.0^{\circ} \mathrm{C}\left(100.4^{\circ} \mathrm{F}\right)$ (normal range, $36.3-37.2^{\circ} \mathrm{C}$ ), a respiratory rate of 20 breaths $/ \mathrm{min}$ (normal range, $16-20$ breaths $/ \mathrm{min}$ ), a heart rate of 80 beats $/ \mathrm{min}$ (normal range, 60-100 beats/min) and a blood pressure of 110/65 mmHg (normal range, 90-140/60-90 mmHg). A chest examination revealed a moist rale and decreased tactile fremitus in the lower left chest. Examination of the head, ears, oral cavity and throat was unremarkable. No swelling of the lymph nodes was detected in the neck.

A complete blood count revealed a white blood cell count of $8.51 \times 10^{9} / 1$, with $81.9 \%$ neutrophils. Chest computed tomography $(\mathrm{CT})$ revealed a $5.6 \times 3.0 \times 4.7-\mathrm{cm}$ mediastinal soft-tissue mass, which had invaded the trachea, a swollen nodule in the upper right mediastinum and pneumonia in the inferior lobe of the left lung (Fig. 1A). Flexible bronchofiberscopy revealed a 4-cm red, granular mass on the posterior tracheal wall that was $5 \mathrm{~cm}$ below the glottis. The mass resulted in $\sim 90 \%$ central airway obstruction, which prevented the passage of a flexible bronchofiberscope through the airway (Fig. 2A). Multiple biopsies of the tumor identified a squamous cell papilloma with moderate to severe atypical hyperplasia and squamous cell carcinoma formation (Fig. 3A and B). The pathological findings of the specimens were confirmed by the West China Hospital of Sichuan University (Chengdu, China). Infection with HPV strains 6, 11, 16, 18, 31, 33, 35, 39, 40, 42-45, 51-54, $58,59,66,68,70$ and 81 in the tumor specimens was ruled out by performing polymerase chain reaction analysis. 

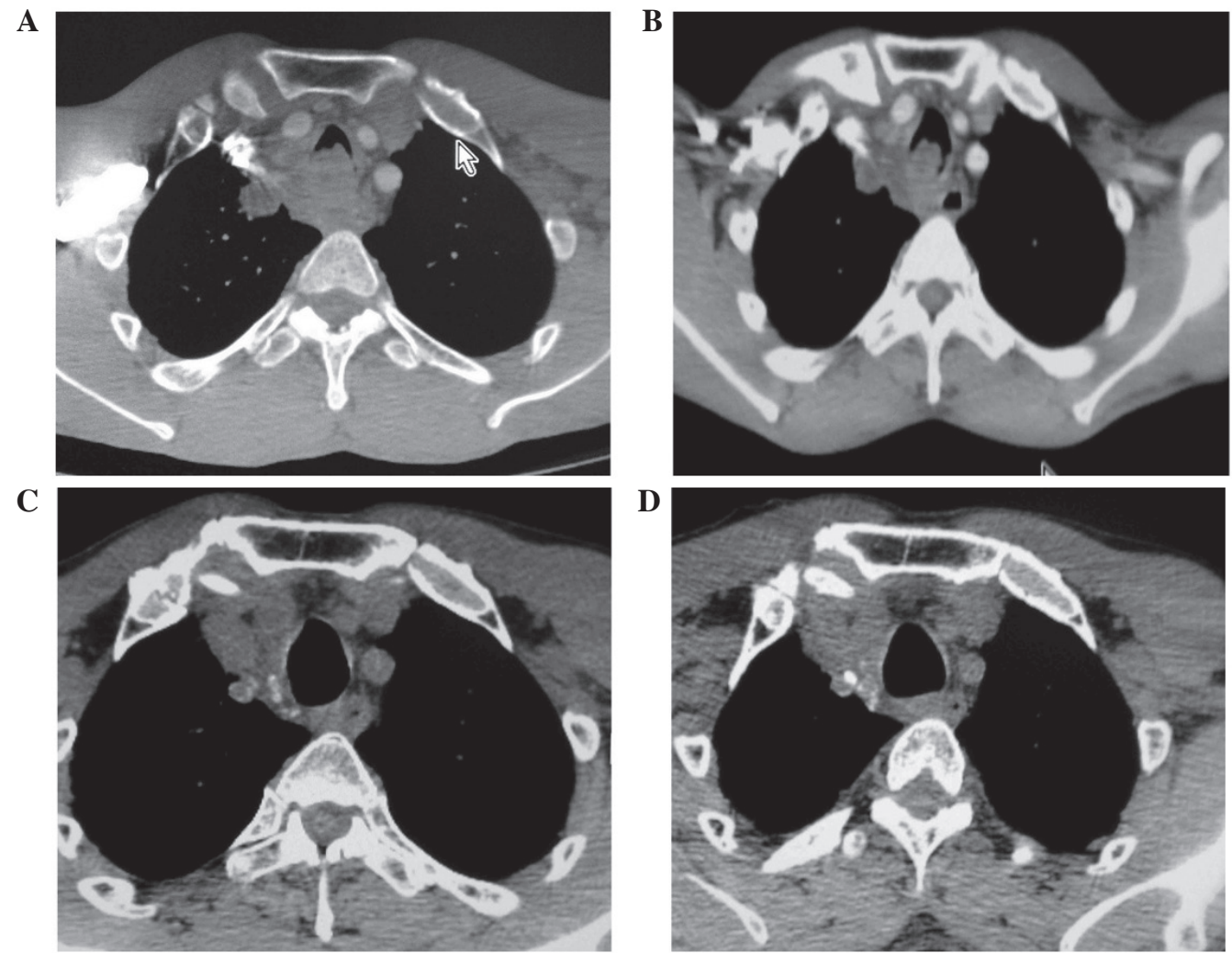

Figure 1. Thoracic computed tomography of the tumor (A) prior to treatment, (B) subsequent to treatment with a radiation dose of $36 \mathrm{~Gy}$, and (C) two and (D) four months after radiotherapy.

A

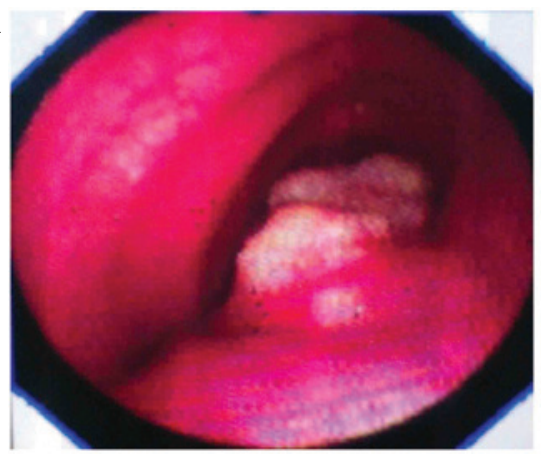

B

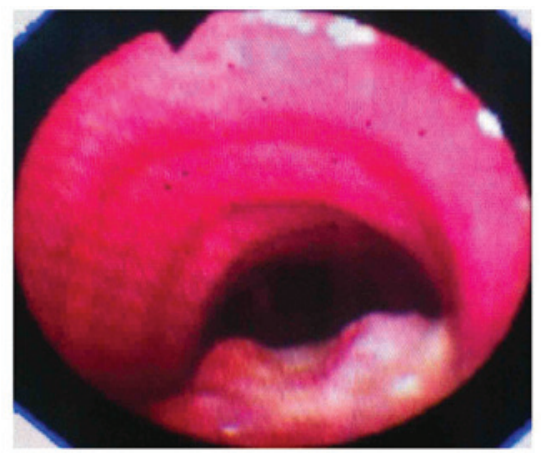

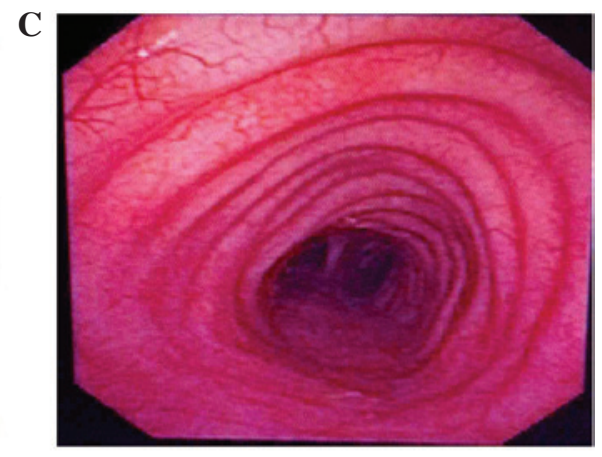

Figure 2. Flexible bronchoscopy of the tumor (A) prior to treatment, (B) subsequent to a radiation dose of 36 Gy, and (C) two months after radiotherapy..

The day after admission, the patient's temperature rose to $39.3^{\circ} \mathrm{C}\left(102.74^{\circ} \mathrm{F}\right)$. In addition, the symptoms of dyspnea were aggravated upon reclining, and the patient had to be kept seated. A sputum culture resulted in Klebsiella pneumoniae growth, and a drug sensitivity analysis revealed that the strain was sensitive to cefotaxime. Therefore, intravenous cefotaxime $(2.0 \mathrm{~g})$ was administered every $12 \mathrm{~h}$ and, after six days, the patient's temperature returned to normal levels.

The case was discussed by a multidisciplinary team that included anesthesiologists, physicians, oncologists and thoracic surgeons, who agreed that the tumor could not be resected successfully due to its invasive growth. Therefore, radiotherapy was administered four days after diagnosis, on August 24, 2011.

Initially, radiotherapy was performed with the patient in a sitting position. Following 18 fractions of radiotherapy ( $2 \mathrm{~Gy} /$ fraction), the symptoms of cough, sputum, dyspnea and hemoptysis were alleviated, and the patient was able to receive radiotherapy in the reclined position without any discomfort. At this time, a CT scan and flexible bronchofiberscopy indicated a decrease in the size of the tumor to $5.4 \times 2.5 \times 4.5 \mathrm{~cm}$ (Figs. 1B and $2 \mathrm{~B}$ ). Therefore, a radiotherapy regimen using three-dimensional conformal radiotherapy was administered in the reclined position for 42 days. The gross tumor volume (GTV) was defined as the volume of visible tumor as determined using the mediastinal window setting. The soft-tissue of the mediastinum, trachea and nodule in the upper right mediastinum were included in the GTV. The clinical target volume (CTV) included the GTV with 5-mm margins along the $\mathrm{x}$ and $\mathrm{y}$ axes, and $15-\mathrm{mm}$ margins along the $\mathrm{z}$ axes. The planning target volume (PTV) included the CTV with a 7-mm margin. The total dose prescription was $60 \mathrm{~Gy} / 30 \mathrm{~F}$ for the PTV. The V20 to the lung was $8 \%$, and the maximal dose to the spinal cord was $4570 \mathrm{cGy}$. During 

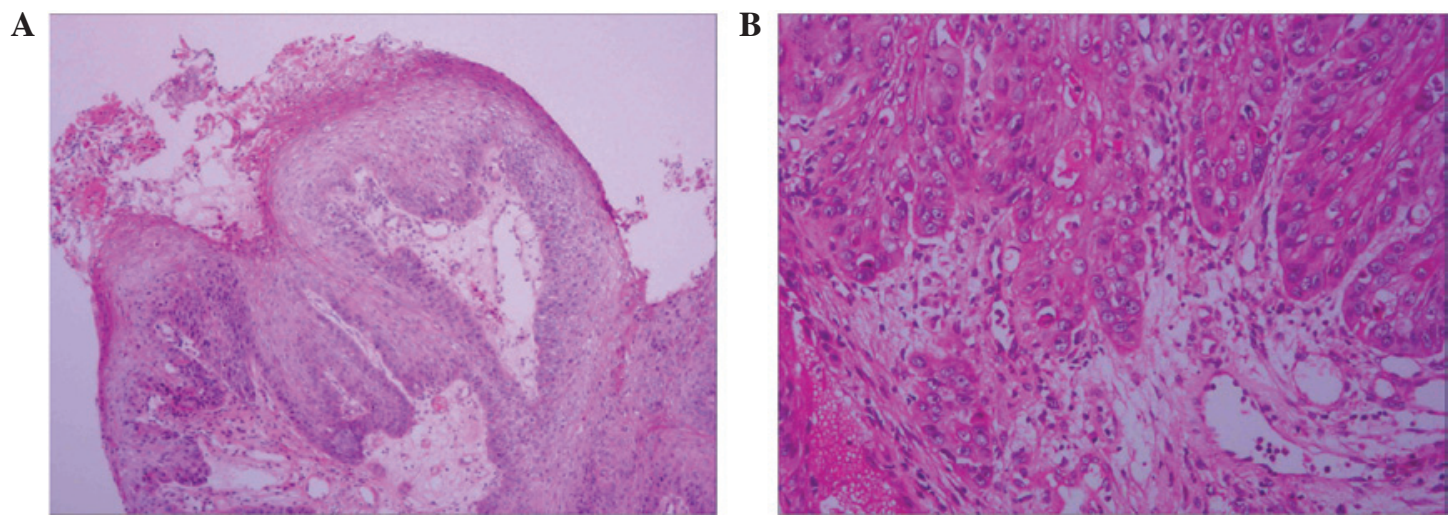

Figure 3. Histological examination revealing papillary growth of the (A) squamous cell papilloma and (B) squamous cell carcinoma formation [stain, hematoxylin and eosin; magnification, (A) x40 and (B) x200].

radiotherapy, the patient experienced moderate pain when swallowing as a result of radiation-induced esophagitis.

A CT scan performed two months after the completion of radiotherapy treatment revealed that the mass in the trachea had markedly reduced in size. The mediastinal soft-tissue mass and the nodule in the upper right lung were significantly reduced, but exhibited slight calcification (Fig. 1C). After four months, routine fiber bronchoscopy indicated no recurrent papilloma at the same site. However, a CT scan revealed further evidence of calcification (Figs. 1D and 2C). The patient demonstrated disease-free survival, with no evidence of recurrence during a two-year follow-up period. At the time of writing, the patient remained alive with no symptoms of disease.

\section{Discussion}

Tracheal papillomatosis is the manifestation of recurrent respiratory papillomatosis (RRP), which usually occurs during early childhood and between the ages of 30 and 40 years (3). However, only a few cases of RRP affecting elderly patients have been reported (3). The incidence of adult RRP in the United States is estimated to be 1.8 cases per 100,000 of the population (6). Despite this, $<50$ cases of solitary endobronchial papillomas have been reported in the English literature (7). Pathologists classify solitary papillomas into three types as follows: i) Squamous papilloma; ii) glandular papilloma; and iii) mixed papilloma. Squamous papilloma is most commonly diagnosed in smokers, aged between 26 and 74 years old (8). Malignant transformation is rare, with a $1 \%$ occurrence rate in cases of juvenile RRP and a 3-7\% in cases of adult RRP (9). Malignant transformation may occur at any location in the respiratory tract; however, transformation in the trachea is extremely rare (10).

Multiple papillomas of the tracheobronchial tree with malignant transformation have been first reported by DiMarco et al (11) in 1978. To the best of our knowledge, only four cases of malignant transformation of a solitary papilloma in the trachea had been reported in the English literature until $2012(12,13)$.

The mechanism of transformation from RRP to squamous cell carcinoma remains unclear. A previous study reported that the majority of malignant transformation cases are associated with HPV type, smoking status, performance of irradiation, immunosuppressant use and exposure to carcinogenic agents (10). The present study described the case of a heavy smoker who presented no evidence of HPV infection. HPV has been associated with the pathogenesis of solitary tracheobronchial papilloma (13). In total, 100 different types of HPV have been identified, each associated with different malignant potentials and symptom severities. HPV 6 and 11 are the most commonly identified types in cases of solitary tracheobronchial papilloma and are associated with a low risk of carcinomatous transformation $(1,13)$. In addition, HPV types 16 and 18, occasionally in combination with types 31,33 or 35 , are associated with a higher risk of carcinomatous transformation (13). Rady et al (14) analyzed the molecular genetic alterations in a case of squamous cell carcinoma that had arisen from RRP. Integrated and episomal forms of HPV 11 DNA have been detected in histologically benign tumors. By contrast, only the integrated form of HPV 11 DNA has been identified in malignant tumors. Furthermore, an allelic loss of the interferon- $\beta$ gene and an endogenous mutation in the p53 tumor suppressor gene have only been detected in malignant tumors (14). A study by Go et al (15), examined the histological and molecular characteristics of seven cases of malignant transformation of RRP to squamous cell carcinoma (three of which demonstrated tracheal involvement). The authors revealed that spontaneous transformation of RRP to squamous cell carcinoma was not characterized by a consistent temporal histological progression from squamous papilloma to severe dysplasia and then to squamous cell carcinoma. HPV 6 and 11 are the most common types of HPV associated with RRP. However, the HPV status may be altered during malignant transformation. HVP types 16, 18, 31, 35 and 51 are rarely identified in cases of RRP and RRP-associated carcinomas (15). Furthermore, the p53 status is not considered to be a molecular marker for malignant transformation and its expression is variable in papillomas and carcinomas (15).

The symptoms of tracheal papilloma are usually attributed to airway obstruction. Patients often present with wheezing, dyspnea, cough, hemoptysis and obstructive pneumonia. Occasionally, tracheal papilloma is masked by other respiratory diseases, such as asthma (16). In all tracheal papilloma patients, including the patient of the present study, the disease is diagnosed by bronchoscopy. In addition, helical CT is a useful tool for determining the location of the papilloma. Squamous cell carcinoma may be difficult to distinguish from papillomas with dysplasia on the basis of cytological specimen findings. 
Therefore, histopathological examination is required (13). Fluorine-18 fluorodeoxyglucose-positron emission tomography (FDG-PET) is more suitable for determining lung cancer staging, and particularly nodal staging, compared with CT. However, to the best of our knowledge, the use of FDG-PET or PET/CT to detect malignant transformation of squamous cell papilloma in the lungs has not been previously reported. Szyszko et al (17) and Pipavath et al (18) detected benign RRP tumors in two patients and identified that benign papilloma and enlarged lymph nodes may exhibit marked FDG uptake. The standardized uptake value (SUV) for lungs is 8.4 , with a maximum SUV of 13. The SUVs for the right hilar and precarinal mediastinal lymph nodes are 4.5 and 5 , respectively $(17,18)$.

Since RRP is a rare disease, large-scale trials concerning RRP-targeted therapies are limited, and the literature mainly includes case reports. RRP in the trachea is primarily treated by surgery and resection techniques, including tracheotomy and bronchoscopy with excision. Other treatments include repeated laser therapy and photodynamic therapy with photosensitizing agents, such as intralesional cidofovir, dihematoporphyrin ether, systemic and intralesional interferon, acyclovir, indole 3-carbinol, cimetidine and retinoic acid (13). In addition, a previous study described the case of a 32-year-old male with tracheal papilloma who was treated with the vascular endothelial growth factor inhibitor, bevacizumab (19). However, the aforementioned treatments are associated with a high incidence of recurrence.

The present study described a rare case of malignant transformation of a solitary squamous cell papilloma in the trachea. To the best of our knowledge, this is the first reported study to treat a patient with this indication using radiotherapy. Following treatment with a radiation dose of $36 \mathrm{~Gy}$, a CT scan revealed a decrease in the size of the tumor from $5.6 \times 3.0 \times 4.7 \mathrm{~cm}$ to $5.4 \times 2.5 \times 4.5 \mathrm{~cm}$. Bronchofiberscopy confirmed the tumor reduction. Furthermore, the tumor was not detectable by CT one month after radiotherapy. These observations indicate that this type of tumor is sensitive to radiation. In addition, the findings of the present study provide evidence for the treatment of RRP of the lung. Unlike the findings of previous studies on non-small-cell lung cancer and RRP of the lung $(7,9,18)$, CT during the follow-up examination revealed calcification in the mediastinum where the swollen nodule used to reside.

\section{References}

1. Shibuya H, Kutomi T, Kujime K, et al: An adult case of multiple squamous papillomas of the trachea associated with human papilloma virus type 6. Inter Med 47: 1535-1538, 2008.
2. Popper HH, Wirnsberger G, Jüttner-Smolle FM, et al: The predictive value of human papilloma virus (HPV) typing in the prognosis of bronchial squamous cell papillomas. Histopathology 21: 323-330, 1992.

3. Harris K and Chalhoub M: Tracheal papillomatosis: what do we know so far? Chron Respir Dis 8: 233-235, 2011.

4. Harman EM and Mosenifar Z: Recurrent respiratory papillomatosis. http://emedicine.medscape.com/ article/302648-overview, 2003. Accessed December 28, 2013.

5. Lott DG and Krakovitz PR: Squamous cell carcinoma associated with intralesional injection of cidofovir for recurrent respiratory papillomatosis. Laryngoscope 119: 567-570, 2009.

6. Armstrong LR, Derkay CS and Reeves WC: Initial results from the national registry for juvenile-onset recurrent respiratory papillomatosis. RRP Task Force. Arch Otolaryngol Head Neck Surg 125: 743-748, 1999.

7. Paganin F, Prevot M, Noel JB, Frejeville M, Arvin-Berod C and Bourdin A: A solitary bronchial papilloma with unusual endoscopic presentation: case study and literature review. BMC Pulm Med 9: 40, 2009

8. Flieder DB, Koss MN, Nicholson A, et al: Solitary pulmonary papillomas in adults: a clinicopathologic and in situ hybridization study of 14 cases combined with 27 cases in the literature. Am J Surg Pathol 22: 1328-1342, 1998.

9. Hasegawa Y, Sato N, Niikawa H, Kamata S, Sannohe S, Kurotaki H, Sasaki T and Ebina A: Lung squamous cell carcinoma arising in a patient with adult-onset recurrent respiratory papillomatosis. Jpn J Clin Oncol 43: 78-82. 2013.

10. Guillou L, Sahli R, Chaubert P, et al: Squamous cell carcinoma of the lung in a nonsmoking, nonirradiated patient with juvenile laryngotracheal papillomatosis. Evidence of human papillomavirus-11 DNA in both carcinoma and papillomas. Am J Surg Pathol 15: 891-898, 1991.

11. DiMarco AF, Montenegro H, Payne CB Jr and Kwon KH: Papillomas of the tracheobronchial tree with malignant degeneration. Chest 74: 464-465, 1978.

12. Lioulias A, Misthos P, Papagiannakis G, et al: Malignant conversion of a solitary papilloma in the distal trachea: report of a case. Can Respir J 7: 375-376, 2008.

13. Lang TU, Khalbuss WE, Monaco SE and Pantanowitz L: Solitary tracheobronchial papilloma: Cytomorphology and ancillary studies with histologic correlation. Cytojournal 8: 6, 2011.

14. Rady PL, Schnadig VJ, Weiss RL, et al: Malignant transformation of recurrent respiratory papillomatosis associated with integrated human papillomavirus type 11 DNA and mutation of p53. Laryngoscope 108: 735-740, 1998.

15. Go C, Schwartz MR and Donovan DT: Molecular transformationg of recurrent respiratory papillomatosis: viral typing and P53 overexpression. Ann Otol Rhinol Laryngol 112: 298-302, 2003.

16. Kim S, Park JK, Park SJ, et al: Solitary bronchial squamous papilloma presenting as a plaque-like lesion in a subject with asthma. Am J Resp Crit Care Med 183: 555-556, 2011.

17. Szyszko T, Gnanasegaran G, Barwick T, et al: Respiratory papillomatosis of lung and F-18 FDG PET-CT. Clin Nucl Med 8: 521-522, 2009.

18. Pipavath SN, Manchanda V, Lewis DH, Schmidt RA et al: $18 \mathrm{~F}$ FDG-PET/CT findings in recurrent respiratory papillomatosis. Ann Nucl Med 22:433-436, 2008.

19. Nagel S, Busch C, Blankenburg T and Schütte W: Treatment of respiratory papillomatosis - a case report on systemic treatment with bevacizumab. Pneumologie 63: 387-389, 2009 (In German). 